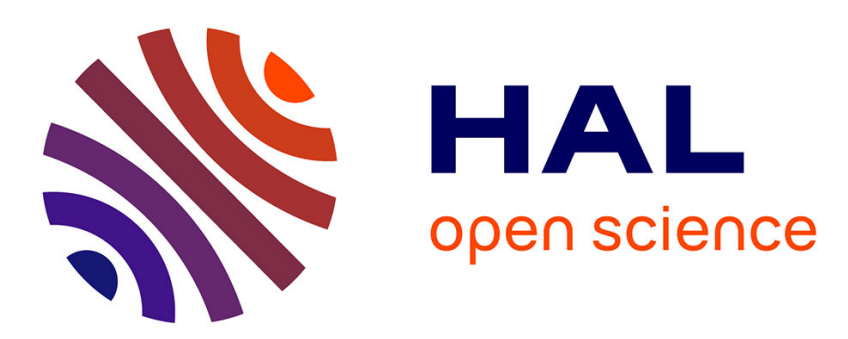

\title{
Des mœurs sexuelles des Sélénites (Lucien, Histoires vraies, I, 22) : entre satire queer et constructionnisme incarné, le sexe qui donne à rire et à penser Michel Briand
}

\section{- To cite this version:}

Michel Briand. Des mœurs sexuelles des Sélénites (Lucien, Histoires vraies, I, 22) : entre satire queer et constructionnisme incarné, le sexe qui donne à rire et à penser. Archimède: archéologie et histoire ancienne, 2018, Archimède ${ }^{\circ} 5$. Archéologie et histoire ancienne, 5, pp.95-107. halshs-01825211

\section{HAL Id: halshs-01825211 \\ https://shs.hal.science/halshs-01825211}

Submitted on 29 Jun 2018

HAL is a multi-disciplinary open access archive for the deposit and dissemination of scientific research documents, whether they are published or not. The documents may come from teaching and research institutions in France or abroad, or from public or private research centers.
L'archive ouverte pluridisciplinaire HAL, est destinée au dépôt et à la diffusion de documents scientifiques de niveau recherche, publiés ou non, émanant des établissements d'enseignement et de recherche français ou étrangers, des laboratoires publics ou privés. 


\section{ARCHIMÈDE N5

DOSSIER THÉMATIQUE : HUMOEROTICA

1 Ruby BLONDELL et Sandra BOEHRINGER

Humour et érotisme dans I'Antiquité grecque et romaine. Introduction au dossier

7 Marina HAWORTH

The Wolfish Lover: The Dog as a Comic Metaphor in Homoerotic Symposium Pottery

24 James ROBSON

Whoring, Gaping and Hiding Meat: The Humour of Male-on-Male Sexual Insults in Aristophanes' Knights

\section{Carmen DAMOUR}

De qui se moque-t-on? Les travestis sur la scène de l'Assemblée des femmes d'Aristophane

49 Deborah KAMEN

The Consequences of Laughter in Aeschines' Against Timarchos

57 Yvonne RösCH

Hunting Hares and Lovers: Socrates' Playful Lesson in Xenophon, Memorabilia III, 11

71 Eugene O'CONNOR

Aroused by Laughter: Martial's Priapic Humor

83 Sandra BOEHRINGER with the artistic collaboration of Marjolaine FOURTON Not a Freak but a Jack-in-the-Box: Philaenis in Martial, Epigram VII, 67

\section{Michel BRIAND}

Des mœurs sexuelles des Sélénites (Lucien, Histoires vraies, I, 22) : entre satire queer et constructionnisme incarné, le sexe qui donne à rire et à penser

108 ACTUALITÉ DE LA RECHERCHE : DES FEMMES PUBLIQUES. GENRE, VISIBILITÉ ET SOCIABILITÉ DANS L'ANTIQUITÉ GRECQUE ET ROMAINE

185 VARIA

246 LA CHRONIQUE D'ARCHIMÈDE

\section{@creative $(1)(9)$}




\title{
DES MCEURS SEXUELLES DES SÉLÉNITES (LUCIEN, HISTOIRES VRAIES, I, 22) : ENTRE SATIRE QUEER ET CONSTRUCTIONNISME INCARNÉ, LE SEXE QUI DONNE À RIRE ET À PENSER
}

\author{
Michel BRIAND \\ Professeur, langue et littérature grecques \\ Université de Poitiers \\ EA 3816 FoReLL \\ michel.briand@univ-poitiers.fr
}

\section{RÉSUMÉ}

Dans les Histoires vraies de Lucien de Samosate, les questions de genre et de sexualité jouent un rôle crucial, comme objets propres à provoquer le rire, tout en donnant à penser. On se concentre ici sur la description des mœurs des Sélénites (I. 22-25), comme dispositif parodique d'inspiration sceptique et cynique, fondé sur le sério-comique (spoudogeloion) et mettant en crise à la fois les discours d'enquête historique, d'anthropologie culturelle et physique et $d$ 'histoire naturelle, par le passage à la limite absurde que provoquent la fiction fantastique et les jeux de langage comiques et sophistiques qui la fondent. On observe ainsi une figure paradoxale du sophiste/philosophe Lucien, constructionniste, relativiste, postmoderne, qui ne manque pas de rappeler

MOTS-CLÉS

Genre,

sexualité, reproduction, Lucien de Samosate, Histoires vraies, sério-comique, scepticisme, satire,

queer, réception. une approche queer, également attachée à I'hybridation, l'extravagance spectaculaire et la pensée critique. L'analyse s'achève par quelques exemples problématiques de réception moderne et contemporaine.
In the True Stories of Lucian of Samosata, the issues of gender and sexuality are crucial, as they efficiently can provoke both laughter and thought. This study focuses on the description of the Selenites and their way of life (I. 22-25), as a parodic device inspired by Scepticism and Cynicism and based on seriocomic effects (spoudogeloion): it puts in a state of crisis historical inquiry, cultural and physical anthropology as well as natural sciences. This crisis is based on fantastic comic fiction and its typically sophistic language plays. Lucian here is paradoxically both a sophistic and philosophical figure, at the same time constructionist, relativist, postmodern: he might remind us of a queer perspective, simultaneously interested in hybridization, spectacular extravagance, and critical thinking. The study ends with some problematic examples of modern and contemporary reception.
KeYWords

Gender, sexuality, reproduction, Lucian of Samosata True Stories, seriocomic, scepticism, satire, queer reception 


\section{POUR UNE POSSIBLE APPROCHE QUEER DES GENRES ET SEXUALITÉS ANTIQUES}

Dans le cadre général des études sur le genre et la sexualité dans I'Antiquité, en particulier dans la fiction, cette analyse d'un épisode singulier des Histoires vraies de Lucien de Samosate, la description des mœurs sexuelles des habitants de la Lune $(I, 22)$, s'inscrit dans une mouvance épistémologique et méthodologique qu'on qualifie de plus en plus souvent de queer. Pour une présentation synthétique de ce terme polysémique, à l'origine injurieux, « bizarre, tordu », à l'égard des personnes LGBT+, qui se le sont réapproprié dans un sens positif, afin de désigner un ensemble de pratiques et représentations, y compris artistiques et théoriques, constitutivement transdisciplinaires, on renvoie à la récente Encyclopédie critique du genre. Corps, sexualité, rapports sociaux, à l'article Queer [1] et à d'autres interrogeant «la chair des rapports sociaux [2] » et des points utiles ici, tels que le genre comme performance et construction, la critique des bicatégorisations simples du type hétéro/homo et femme/ homme, I'intersectionnalité genre/race/classe mais

[1] Voir, dans Rennes (dir.) 2017, Maxime Cervulle et Nelley Quemener, «Queer », p. 529-538, ainsi que Keivan Djavadzadeh, «Culture populaire » (p. 183191), Luca Greco et Stéphanie Kunert, « Drag et performance $\gg($ p. 222-231), Sébastien Chauvin et Arnaud Lerch, « Hétéro/homo » (p. 306-320), ou Anne Creissels, «Mythe/métamorphose » (p. 390-399). Dans cet ouvrage non exhaustif, qui relève des sciences sociales, les études littéraires (et la psychologie) sont peu présentes, mais les auteurs, comme il est habituel dans les études de genre, s'intéressent aux notions de discursivité, performativité, énonciation, subjectivation ou incorporation, pour lesquelles les études sur les littératures anciennes se sont révélées utiles : FouCAULT 1984 s'intéressait, comme à des documents, aussi aux littératures grecques d'époque impériale, en particulier au roman grec, contemporain de Lucien, même s'il ne mettait pas en valeur leur dimension fictionnelle et comique. Voir aussi Holmes 2012, en particulier « Butler and Beyond : the Future of Gender », p. $168-180$ et « Conclusion », p. 181-183. Sur le style, les aussi apparence physique/âge/santé. S'agissant des études dites classiques, en domaine francophone mais aussi anglophone, souvent des ouvrages importants, à visée scientifique, critique et pédagogique, ne se réfèrent pas aux études queer nommées comme telles, tout en en étant proches, en termes de constructionnisme, relativisme et fluidité des catégories, généalogies critiques et multi-dimensionnalité des identités, dangers de l'essentialisme et de l'ethnocentrisme, ou intérêt pour une «pratique contrôlée de l'anachronisme [3] » et du « double écart comparatif [4] », en même temps que pour la dialectique entre actualité et inactualité, ou « intempestivité [5] » : il est vrai que le développement des études de genre a rencontré des oppositions institutionnelles, académiques et sociales vives, favorables à une certaine prudence terminologique, que I'analyse présentée devrait pouvoir plus aisément dépasser, désormais. On insiste aussi sur le fait que les antiquisants spécialistes des questions de genre et sexualité évoquent volontiers des cultures « d'avant la sexualité », pour lesquelles de nombreuses catégorisations modernes, souvent nées au XIX ou $\mathrm{XX}^{\mathrm{e}}$ siècles, ne sont pas opératoires. Sans dire que

objectifs et objets d'étude de la pensée critique queer, dans le domaine des arts, un bon exemple est MoINEAU 2016.

[2] p. 24.

[3] Voir LORAUX 2005 (1993).

[4] Sur la notion d'écart, voir aussi les activités de l'association Antiquité Territoire des Écarts (https://labantique. hypotheses.org).

[5] Sur le rapport antique / contemporain, voir BRIAND 2012, 2015, 2016. Sur des études de genre qui, sans souvent se référer explicitement à une théorie queer, s'en approchent, voir BoEHRINGER \& SÉBILLOTTE (dir.), 2010, BoEHRINGER \& BRIAND 2012, BoEHRINGER \& LORENZINI 2016, ou HUBBARD (dir.) 2013 et MASTERSON, RABINOWITZ \& ROBSON 2015. Sur le caractère crucial, y compris politiquement, de ce type de généalogie, voir NARBONNE 2016, chap. IV « Du scepticisme ancien à la tolérance moderne : I'héritage paradigmatique de Lucien », p. 139-189. 
la théorie et les pratiques queer seraient à situer « après la sexualité », on note qu'elles tentent de troubler ces mêmes catégorisations modernes et que donc une rencontre est possible ici, dans une perspective critique.

Mais on ira plus loin encore, en se référant à ce que, d'abord dans les études de réception, on peut appeler queer classics, par distinction à des straight classics, correspondant à une conception plus linéaire, parfois plus positiviste, de I'histoire de la littérature et de la philologie [6]. Deux aspects interdépendants peuvent entraîner la qualification de queer :

- dans la perspective d'un écart psychologique, éthique et social, «sexuel », une production culturelle ancienne, par le contenu thématique qui s'y trouve présenté et du style qui s'y déploie, par exemple avec un intérêt marqué pour les questions de genre et de sexualité non binaires, non conformes, hybrides, etc, et par son usage de spectaculaires effets rhétoriques et poétiques combinés avec un goût affirmé pour le mélange des genres discursifs et littéraires et la combinaison de lectures à la fois fortement empathique et critique. Lucien, notre auteur de fictions à la fois sensationnelles et cultivées, a pu être dit « postmoderne », à l'instar d'un Umberto Eco, sémioticien auteur de best-sellers [7], et, par certains aspects, son humour peut avoir quelque chose à voir avec la notion moderne $d^{\prime}$ « humour camp », indissociable du queer, une sorte de « kitsch assumé », très fréquent, entre autres, dans certains usages contemporains des références à l'Antiquité, par exemple dans la culture dite pop [8].

- dans la perspective d'un écart critique, la position du critique moderne analysant les productions anciennes, qu'elles soient ainsi qualifiables ou non. Il s'agit d'un regard « de travers », combinant, comme chez Lucien, lecture immersive et distance critique, voire ironique, engagement et réflexivité, intérêt combiné pour le contexte de création des objets culturels étudiés (textes, images, concepts) et les enjeux de toutes les réceptions, réécritures, réélaborations, usages qu'ils ont connus. On s'approche ici d'un postclassicisme [9], par lequel la théorie critique contemporaine peut aider à revivifier le travail philologique, et inversement, et la relation entre antiquité et contemporanéité.

Dans les deux cas, objet et regard sur cet objet, la question du rapport entre rire et sexe dans I'Antiquité est cruciale, comme matériau ancien et catalyseur d'études classiques proprement contemporaines. Mais le rire étant susceptible d'analyses aussi constructionnistes, relativistes, dans le temps et l'espace, et transdisciplinaires que le genre et la sexualité, on a préféré se concentrer sur une modalité spécifique, dans un contexte singulier, chez Lucien de Samosate, représentatif de la culture antique d'époque impériale, lié à la Seconde Sophistique et au roman grec, et polygraphe virtuose doté d'un humour rhétorique, poétique et philosophique empreint de feintise et de paradoxe : ce type de texte n'est pas un pur document historique, si jamais d'ailleurs cela existe, ou plus exactement I'historien des idées et représentations culturelles, comme à plus forte raison I'historien de la littérature, devra tenir grand compte des dispositifs pragmatiques auxquels participe ce type d'énoncés, surtout quand ils relèvent, comme ici, d'une visée à la fois comique et sérieuse [10].
[6] MATZNER 2016, sur le dialogue entre antiquité impériale et postmodernité queer, dans les romans de José Luis de Juan (Este latente mundo, 1999, trad. fr. Les souffles du monde, 2001) et Jeremy Reed (Boy Caesar, 2004).

[7] Voir Ni Mheallaigh 2014, Lucian's Promethean poetics : hybridity, fiction and the postmodern, p. 1-38, et Conclusion. Fiction and the wonder-culture of the Roman empire, p. 261-277, ainsi que LAMI \& MALTOMINI 1990, Un antico postmoderno, p. 1-35. On renvoie auss à LÜTHE 2013, pour une définition de la postmodernité par l'ironie sceptique, teintée de mélancolie, à notre sens proche de ce qu'on trouve chez Lucien.

[8] Voir BRIAND à paraître 2018 et les activités du groupe Antiquipop (http://antiquipop. hypotheses.org).

[9] Voir par exemple le réseau Postclassicisms (http:// www. postclassicisms.org).

[10] La littérature sur Lucien est trop riche pour être développée ici. On renvoie à BOMPAIRE 2000 (1958) et à des recueils récents comme LIGOTA \& PANIZZA 2007 (dont, pour notre étude, Mauro Bonazzi, « Luciano e lo scetticismo del suo tempo », p. 37-48; Baudouin Descharneux « Lucien doit-il être rangé dans la boîte des philosophes sceptiques ? », p. 63-71; Isabelle Gassino, « Par-delà toutes les frontières : le pseudos dans les Histoires vraies de Lucien », p. 87-98; et Karen Ni Mheallaigh, « The Game of the Name : Onymity and the Contract of Reading in Lucian », p. 121-132), ou plus ancien mais très utile comme BILLAULT 1993/1994 (dont, pour notre étude, Sandrine Dubel, « Dialogue et autoportrait. Les masques de Lucien », p. 19-26 ; Geneviève Husson, « Lucien philosophe du rire ou Pour ce que rire est le propre de I'homme », p. 177-184 ; Suzanne Saïd, « Lucien ethnographe », p. 149-170; et Jean Schneider, « Les scholies de Lucien et la tradition paroemiographique », p. 191-204). Par manque de place, on mentionne aussi des études précédentes, BRIAND 2007, 2008, 2009, 2010, 2015a, 2016, 2017. Voir aussi EISSEN et BRIAND 2014 (dont Michel Briand, « La fiction qui pense en riant : avatars et paradoxes du muthos et du pseudos chez Lucien » ; Ariane Eissen, « Lucien, personnage de fictions lucianesques » ; Marie-Hélène Garelli, « La Danse de Lucien : défense d'une cause ou trait d'esprit ? » ; JeanPhilippe Guez, « Lucien, I'ivresse ou la gueule de bois »; Sophie Rabau, « Pourquoi dit-on que Lucien est un auteur de Science-Fiction? »), et BRIAND, DUBEL \& EISSEN 2017. 


\section{LE SPOUD(AI)OGELOION DE LUCIEN : LES ENJEUX PARADOXAUX DE L'EXTRAVAGANCE}

En effet, la modalité comique la plus répandue dans les quatre-vingts œuvres attribuées à Lucien est celle, débattue, du spoudogeloion (ou spoudaiogeloion), comico-sérieux ou sério-comique [11]. D'origine cynique, ce registre discursif serait surtout efficace en situation de dialogue philosophique et typique du genre de la satire [12]. Lucien se dit en effet I'inventeur du dialogue comique comme genre autonome, hybridant dialogue socratique et comédie, carnavalesque et utopique, comme dans la comédie ancienne d'Aristophane, ou plus dramatique, éthique et sociale, comme dans la comédie nouvelle de Ménandre. Ce type de rire soutient la parrhêsia ou «liberté de parler », caractérisant des personnages cyniques comme Diogène et Ménippe ou des multiples masques autofictionnels de Lucien [13], Loukinos, le Syrien, ou encore Parrhêsiadês, le « Libre-parleur » [14]. On appréciera donc son ton volontairement provocateur par son intégration dans une pragmatique constante du double sens, de I'indécidabilité éthique, épistémique, aléthique. La préface des Histoires vraies, critique à l'égard des enquêtes historiographiques et géographiques d'Hérodote ou Ctésias, qualifiées de mensongères, s'achève sur une proclamation ambivalente, en forme de contrat métafictionnel [15] : après avoir dit son intention d'offrir un divertissement à la fois populaire et lettré, « un spectacle non sans Muses » (par.

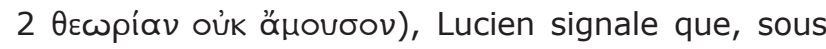
« la bigarrure des fictions rapportées sous une forme

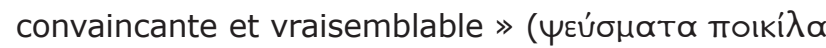

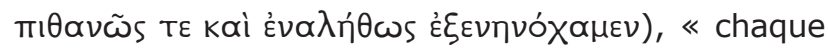
élément de ces histoires est une allusion, non sans

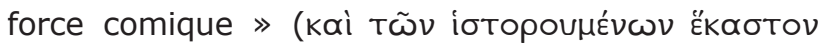

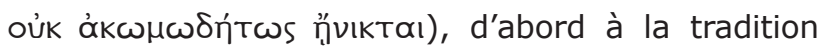
poétique, historique et philosophique. L'auteur de cette préface inouïe, en fait sérieuse, affirme enfin sa

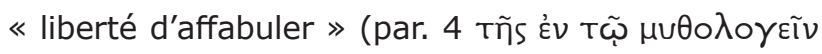

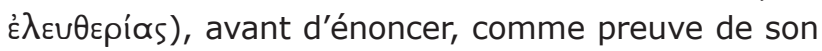
authentique honnêteté : « car vraiment sur ce seul point je serai véridique, en disant que je mens / je

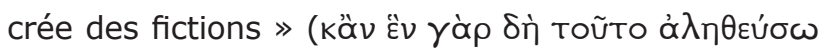
$\lambda \varepsilon ́ \gamma \omega \nu$ öTı $\psi \varepsilon u ́ \delta o \mu \alpha ı)$. Cela concerne aussi tout discours ou récit, du narrateur ou d'un personnage, sur les questions de genre et de sexualité. Au début de sa description des mœurs sélénites (I, 22), Lucien

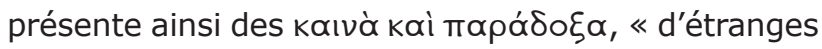
extravagances », et pour rendre l'idée de paradoxe, « contraire au sens commun », « absurde», à la fois figure de rhétorique et forme de raisonnement philosophique, on utilisera la notion $d^{\prime}$ « extravagance »[16], avec ses modalités spectaculaires, comiques et fantastiques, et le fait qu'on ait là, chez Aristophane comme chez Lucien, une « manière de penser », voire un « système de pensée » et un « mode d'écriture ». On ajoute qu'il s'agit de «penser en riant », et donc de «penser le genre et la sexualité en riant », en décrivant des fictions surprenantes, voire aberrantes, qui permettent, par écart, de mieux apprécier et critiquer des évidences contemporaines, du temps de Lucien comme du nôtre [17].

\section{SEXES, GENRES ET SEXUALITÉS : LE CAS-LIMITE DES SÉLÉNITES (I, 22)}

Le premier livre des Histoires vraies est occupé par deux épisodes, le séjour sur la Lune, centré sur la guerre avec le royaume du Soleil puis sur une anthropologie des Sélénites, et le séjour à l'intérieur
[11] Voir HALLIWELL 2008, « Lucian and the laughter of life and death », p. 429-469, et « What made Cynics laugh?», p. 372-387 ; BRANHAM 1989 et 2004 (rapprochant Lucien et Nietzsche, par leur goût pour le scepticisme et le cynisme, la mise en récit et en scène d'expériences de pensée philosophique et l'humour) ; dans une perspective littéraire et sociologique, ANDERSON 1976 ; et, plus lié à une pensée critique contemporaine, WhITMARSH 2011, chap. V « Satirizing Rome : Lucian», p. 247-293, et 230, chap. 15, « Imagine », p. 215-230. Sur le spoudogeloion en général, voir GIANGRANDE 1992, et, chez Aristophane, ERCOLANI 2002.

[12] Voir HELMER 2017.

[13] L'expression « masques » est empruntée à S. Dubel (BILLAULT 1993/1994, voir note précédente). Une partie de la théorie littéraire contemporaine voit en Lucien un des précurseurs, voire inventeurs, de l'autofiction, cf. ColonNA 2004 (« Lucien le Magnifique », p. 21-66 et
« L'autofiction fantastique », p. 75-92, avant les postures autofictionnelles dites biographique, spéculaire et intrusive).

[14] Sur la parrhêsia chez Lucien, voir BRIAND 2016 (et I'avant propos « "Maintenant donc pour des mots vous allez me tuer?", Lucien de Samosate. Du blasphème en démocratie, par un détour en Grèce ancienne », dans Céline Lageot \& Fabien Marchadier (dir.), Le blasphème dans une société démocratique, Dalloz, Paris, 2016, p. 11-17) ; CAMEROTTO 1998 et surtout 2014 (chap. IV «Le virtù e la potenza della vista », p. 191-224, et V «La libertà e il dovere del dire », p. 225-283); et, dans toute I'Antiquité, SLUITER \& ROSEN 2004, ainsi que, dans une perspective générale, FOUCAULT 2009.

[15] BRIAND 2005.

[16] ]AY-ROBERT 2015.

[17] EISSEN \& BRIAND 2014. 
d'une baleine, aussi guerrier et ethnographique. Dans les deux cas, on assiste à un déplacement spatial propice à un écart de perspective, dans une intention sério-comique à la fois satirique et allégorique : la baleine a quelque chose de la Caverne de Platon [18]. Ce point de vue est décentré, parfois surplombant, pour le narrateur et le lecteur, comme dans les Dialogues des morts, une « littérature de l'ailleurs » qui aide, surtout par le rire, à prendre distance avec l'ici-bas, et à le critiquer, déconstruire ou du moins relativiser [19].

Voici le paragraphe principal (I, 22) relatant les coutumes des Sélénites en ce qui concerne le genre, le mariage et la reproduction, dans un style parodiant I'historiographie ethnographique d'Hérodote et Ctésias, la paradoxographie des récits de voyages au long cours à tendance utopique, comme les Merveilles d'au-delà de Thulé (Tà

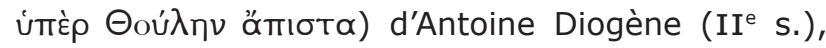
ou encore des histoires naturelles d'inspiration aristotélicienne [20] :

[22] « Ce que durant mon séjour sur la Lune j'ai observé d'inouï et d'extraordinaire

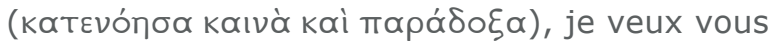
en parler. D'abord, ce n'est pas de femmes qu'ils naissent (

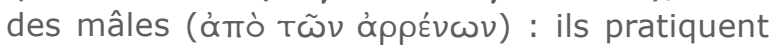

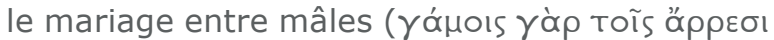

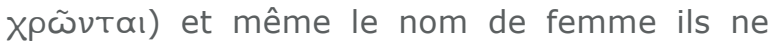
le connaissent pas. Jusqu'à vingt-cinq ans, chacun y est épouse ( $\gamma \propto \mu \varepsilon i \tilde{T} \alpha \mathrm{l})$, et à partir de cet âge à son tour époux $(\gamma \propto \mu \varepsilon \tilde{)})$ : ils sont enceints non pas dans le bas-ventre mais dans

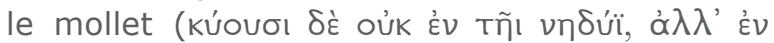

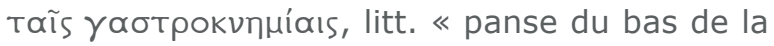
jambe ») ; en effet, quand l'embryon prend, le mollet s'épaissit et un temps plus tard ils l'incisent pour l'en retirer mort et l'exposer au vent, la bouche ouverte, afin de lui rendre la vie. Il me semble que chez les Grecs de là est venu le nom gastrocnémie, parce que chez eux (les Sélénites) au lieu du ventre c'est là qu'on

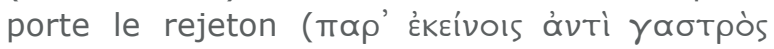
киофорєі̃). Mais je vais raconter quelque chose de plus fort encore : il y a chez eux une espèce

[18] LAIRD 2003. Sur Lucien comme philosophe rieur, voir SETEMBRINI \& FUSARO 2007, « Saggio introduttivo di Diego Fusaro », p. 5-96.

[19] L'une des premières études mettant explicitement en relation la sexualité dans les épisodes des Sélénites et des Femmes-vignes et la lecture (méta)fictionnelle comme parcours similaire à un voyage d'exploration est LARMOUR 1997. Les commentaires des Histoires vraies les plus complets, surtout sur les questions d'hommes, ceux qu'on appelle « Arborigènes » (Dendrites) [21]. Voici comment se fait leur genèse : on coupe le testicule droit d'un humain et on le plante en terre (фuTEúovoıv) ; de là un arbre pousse (ávaqúctal), très haut, fait de chair, comme un phallus; il a des branches et des feuilles et le fruit en est des glands d'une coudée de long (environ 0,44m). Quand ils sont bien mûrs, on les récolte et on les casse pour en extraire les humains. Mais ils ont des parties sexuelles ( $\alpha i \delta$ ioĩ) postiches, certains en ivoire, d'autres, les pauvres, en bois, et c'est avec cela qu'ils font leurs saillies et s'accouplent avec

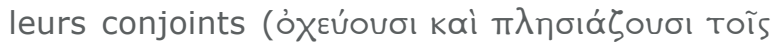

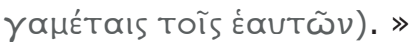

Le comique repose ici sur des procédés conjoints structurant I'ensemble des Histoires vraies et relevant d'une grammaire transformationnelle qui subvertit les genres textuels parodiés :

- inversion, par exemple du rapport genré mâle/ femelle, reporté sur une dissymétrie d'âge, rendue possible par le jeu des diathèses active et passive du verbe $\gamma \propto \mu \omega \tilde{\omega} / \gamma \propto \mu$ oũ $\alpha$, qui ne présente pas en luimême de spécifications formelles de genre : c'est le sujet syntaxique qui permet de préciser ce point et I'assimilation régulière entre sujet féminin et passif, dans le cadre du mariage, est ici troublée, puisqu'un sujet masculin jeune peut l'être du verbe au passif. Le jeu entre voix verbale et relation institutionnelle et sexuelle vise à faire rire, par son aspect contredoxique, mais il montre surtout que cette situation extravagante est autorisée par le langage et donc possible dans une réalité éloignée dans le temps et l'espace ou encore dans la fiction fantastique. Ce type de mariage peut être dit et donc pensé, mais fantastiquement.

- expansion et amplification, par exemple pour l'arbre-phallus et ses glands, longs d'une coudée. Cette figure comique et fantastique à la fois est fréquente dans les Histoires vraies, par exemple pour l'épisode du nid d'alcyon, au périmètre de soixante stades (près de onze kilomètres, II, 40) ou la description épique des batailles cosmiques entre les peuples de la Lune et du Soleil (I, 12-21), opposant des centaines de milliers d'ennemis. Cette

d'intertextualité, sont GEORgIADOU \& LARMOUR 1998 et MöLLENDORF 2000, ce dernier prenant en compte une lecture lettrée, moins sensible à toutes les variétés de comique.

[20] Les traductions sont miennes, souvent inspirées de celles de Chambry, révisées par BILLAULT \& MARQUIS 2015, et d'OZANAM 2009, qui reprend BOMPAIRE 1998.

[21] La traduction « arborigène » est une trouvaille de LACAZE 2003, p. 245. 
forme de comique, comme la précédente, participe du caractère carnavalesque de l'utopie, fondée sur l'exagération d'un trait typique de la « réalité » dont on fait la satire.

- construction d'un monde possible à partir de jeux de langage, comme la physiologie sexuelle des Sélénites, tirée, par extension et associations d'idées, du terme anatomique non sexuel désignant le «mollet», ou gastroknêmia. D’où le fait que le jeu de mots est à peu près impossible à rendre en français et qu'il était plus directement comique pour le lecteur grec. Le caractère paradoxalement réaliste de la description est renforcé par le fait que le narrateur n'évoque pas la manière dont il a procédé, mais explique, dans une démonstration de type scientifique, la terminologie grecque par ce qui se passe sur la Lune, et non l'inverse. Cette procédure revient à parodier les explications étymologiques et étiologiques appliquées à l'anthropologie physique et culturelle et à suggérer que c'est le langage et la fiction qui construisent un monde, par le regard qui l'observe et en dénomme les éléments constitutifs.

- hybridation, surtout du rapport animal/végétal, pour les Dendrites ou «Arborigènes »: le testicule droit d'un Sélénite peut devenir une graine d'arbre et les humains qui en naissent, la chair de fruits à coque. L'öpxis est aussi une plante à bulbe [22] ou une espèce d'olive [23], et la taxinomie moderne a les orchidées du genre orchis. Le ßád $\alpha$ vos est le « gland», fruit du chêne, mais aussi du palmier dattier, chez Hérodote. Il s'intègre dans des matrices métaphoriques dont Lucien exploite les connotations anatomiques, médicales, zoologiques : le « gland du pénis », comme en français [24] ; un « suppositoire » ou « pessaire », chez Hippocrate ; le «pêne » d'un verrou; des animaux marins, mollusques ou crustacés, tels le « gland de mer » ou la balane, chez Aristote aussi. Enfin I'« arbre très haut » des Dendrites est « fait de chair », « comme un $\phi \propto \alpha \lambda \lambda$ ós », représentation du pénis en érection, par exemple en contexte cultuel dionysiaque. Dans les paragraphes précédents ( $I, 11-20$, en particulier I, 13 et 16), les forces guerrières en présence mettent en scène une hybridation de niveau cosmique, à l'intérieur de et entre les ordres de I'humain, de l'animal, du végétal, voire de l'élémentaire : ainsi pour les Hippogypes (« vautours - chevaux » ou « chevautours »[25]), Lachanoptères («ailes de légume »), Anémodromes (« coureurs de vents »), Strouthobalanes (« glands de moineaux »), Aérocordaces («bouffons aériens », fantassins frondeurs, lanceurs de raiforts géants enduits de mauve, à connotation sexuelle et scatologique) [26] et Cynobalanes (« glands chiens », hommes à visage de chien, montant des glands ailés), là aussi riches en connotations soutenant la force comique et la coloration sexuelle, à la fois génitale et anale, du passage.

- enfin, fragmentation, par exemple du corps comme intégrité organique, muni, pour les parties sexuelles, de prothèses artificielles, en ivoire ou bois. C'est aussi la distinction binaire corps naturel/ artificiel qui est troublée, hybridée, comme dans I'ensemble du récit. Plus loin, par. 25, les Sélénites ont des «yeux amovibles », qu'on peut ranger quand on n'en a pas besoin : ici aussi, la perspective est intersectionnelle, certains, ayant perdu leurs yeux, doivent en emprunter, et les plus riches en ayant « de nombreux en réserve ».

Ce dernier point peut subsumer les précédents ; les procédés à l'œuvre dans cette création comique reviennent à faire dépendre la réalité observée du langage et du regard porté par l'observateur : l'objet observé est une construction, non pas un donné. C'est ainsi du nom des Dendrites que procèdent leurs traits évoqués ensuite ou du terme anatomique gastroknêmia que dérive le processus reproductif inventé par le narrateur anthropologue ou « enquêteur ». Tout se présente explicitement comme une fiction comique, mais dans le cadre d'un univers en fait similaire au nôtre, où tout est de toute façon fiction et feintise, comme le proclamait déjà la préface, sous l'égide notamment de I'Ulysse homérique et de ses multiples rencontres prodigieuses, telles qu'il les a lui-même racontées, comme Lucien ici $(I, 3)$.
[22] Théophraste, Histoire des plantes, IX, 18, 3. Pour ces emplois techniques, on se réfère à CHANTRAINE 1999. [23] Columelle , V, 8.

[24] Aristote, Histoire des animaux, I, 13, 3.

[25] Selon la proposition de I'un des deux relecteurs anonymes de la revue.

[26] Les connotations du ṕáqavos ou ṕaqavís « raifort », complétées par celles de la mauve, aux vertus réputées laxatives dans I'Antiquité, sont aussi sexuelles que celles du gland, et plus violentes : la pénétration anale par un raifort est le châtiment des $\mu$ ox oi (« adultères » ?) pris en flagrant délit, dans l'Athènes classique, selon Aristophane (Nuées 1079-1083, où les condamnés

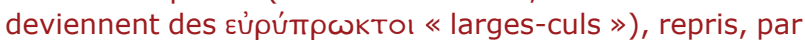
Lucien, Sur la mort de Peregrinos, 9 (où le futur faux pro-

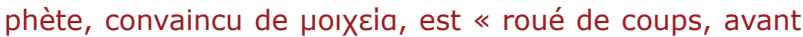
de s'enfuir en sautant d'un toit, un raifort enfoncé dans

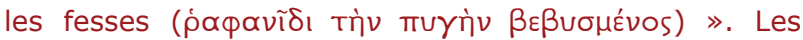
Sélénites ont d'ailleurs une queue en raifort (par. 23). 


\section{UNE INTERSECTIONNALITÉ} FANTASTIQUE : ÂGE, CLASSE SOCIALE, ASPECT, ALIMENTATION (I, 23-25)

La suite de la description (I, 23-25) confirme cette posture constructionniste et en même temps spectaculairement comique, renforcée par le fait que ces textes faisaient d'abord I'objet de performances oratoires où la réception du public, plutôt que du lectorat, dépend de l'actio et de la diction du sophiste, d'autant que le récit est présenté à la première personne du singulier, comme un témoignage personnel. Les questions de genre et de sexualité sont mêlées, comme à la fin du par. 22, à d'autres considérations physiques aux importantes implications culturelles et économiques, ici réordonnées :

- «Ils n'urinent pas ni ne vont à la selle, car ils

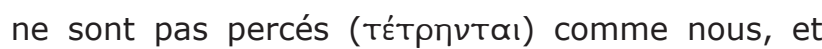
pour les relations sexuelles les garçons n'offrent pas leur fondement mais le pli du genou au-des-

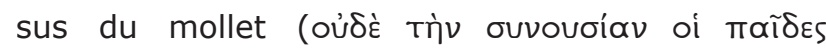

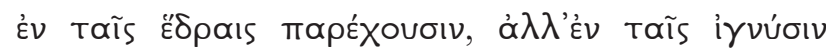

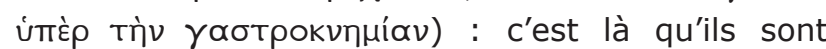

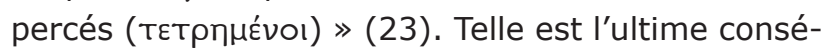
quence du sens littéral assigné au nom grec du « mollet », par un passage à la limite devenu expé-

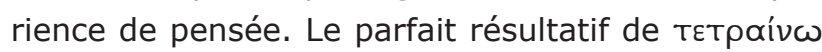
« percer, faire un trou » participe à la parodie des mythes de création des humains, parmi lesquels, dans le Banquet platonicien, celui des trois types de boules humaines doubles raconté par Aristophane, au caractère burlesque parfois sous-estimé [27].

- les allusions comiques, surtout quand elles ont une couleur obscène, peuvent être moins claires pour les modernes que pour les Grecs, de même que le lien entre rire et sexe. Un exemple en est un indice anatomique qui fait des Sélénites une société de Molxoí, « dépravés » (《 adultères », et « mécréants » en grec néo-testamentaire), que I'Athènes classique aurait condamnés au châtiment infâmant du raifort dans I'anus, selon Aristophane [28], alors que sur la Lune, non seulement les pratiques sexuelles décrites ne sont pas condamnables mais elles relèvent de la norme, et «au-dessus des fesses (úmè $\delta \dot{\varepsilon}$ Tàs muyàs), à chacun d'entre eux a poussé un long raifort [29],

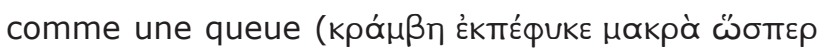
oủpó), vert en permanence et qui, quand on tombe en arrière, ne se casse pas. » L'utopie s'oppose ici au modèle législatif classique, tout en riant d'elle-même.

- symétriquement au nourrisson, mort à la naissance et ramené à la vie par l'action du vent, le vieillard mort « comme une fumée se dissout et devient de l'air » (23). Les Sélénites se nourrissent de la fumée des grenouilles grillées et boivent de l'« air comprimé dans une coupe » (23). Leur morve est « un miel très âcre » et leur sueur du lait, dont ils se nourrissent aussi, et les vignes produisent de l'eau, tout en causant les chutes de grêle sur la Terre (24). « Le vêtement des riches est en verre souple, celui des pauvres en tissu de cuivre », humidifié et travaillé comme de la laine (25). Le mode d'alimentation aérien semble rendre inutiles les intestins : leur ventre est un sac, où on transporte ce qu'on veut; son intérieur est velu, « de sorte que les nouveau-nés, quand ils ont froid, peuvent s'y glisser (24). »

- le relativisme de Lucien s'exprime à propos de la chevelure et des critères de beauté, variables selon le contexte, et inversés sur la Lune, par rapport à la Terre (23) : «On est considéré comme beau chez eux si I'on est chauve et sans cheveux (ä́kopos), et les chevelus (кouńtas) sont en horreur ». Inversement, « dans les comètes » (en grec, «les

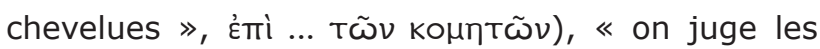
chevelus (кouńtas) beaux ». Mais le narrateur ne procède pas ici par observation directe, autopsie, mais d'après des témoignages de voyageurs. En matière de jugement esthétique, social et moral, tout est affaire d'opinion, dans le vaste monde.

Comme l'indique la préface, parmi de nombreux usages lucianesques du spoudogeloion, le but de cette fiction comique est sérieux : il s'agit, par le plaisir du divertissement même, de faire comprendre au lecteur, et apprécier, des vérités sur la culture grecque, historiographique, scientifique, littéraire, comme sur la notion même de fiction rhétorique et poétique, mais aussi historique ou philosophique, et
[27] Sur les références comiques de Lucien au Banquet de Platon, voir BOEHRINGER \& BLONDELL 2014, ainsi que de nombreux travaux sur le Banquet ou Les Lapithes de Lucien, dernièrement SCOLAN 2017, en particulier « Le banquet au matin et au soir de la philosophie » (p. 111145 ) et «Bas les masques : hypocrites et imposteurs au banquet » (p. 247-287), et la communication présentée au colloque Dévorer/dépenser dans le monde hellénistique et romain (Tours 30-31 mars 2017), BRIAND,
« Voracité et avidité dans Le Banquet ou Les Lapithes de Lucien : enjeux esthétiques et philosophiques », à paraître dans les Actes.

[28] Voir note 25.

[29] Le nom kpó $\mu \beta \eta$ désigne d'abord un « chou », aux

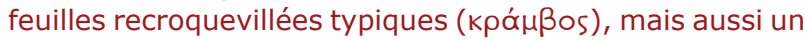
« raifort », chez Aristote (Histoire des animaux, V, 19,4) et Athénée (34d). 
donc, finalement, sur ce qu'on considère, pour aller vite, comme la réalité de notre monde, éminemment digne de satire. C'est ce qui apparaît ensuite, à propos du « Miroir de la Lune » $(\mathrm{I}, 26)$, métonymie de l'ensemble des Histoires vraies.

\section{LE « MIROIR DE LA LUNE » : INVERSIONS, ÉCARTS, STRATES, SURPLOMBS}

Le caractère métafictionnel et critique du dispositif est renforcé par l'image concluant cette enquête imaginaire, le miroir-puits qui permet d'entendre et voir ce qui se passe sur la Terre, dans une inversion jubilatoire, faussement modeste comme souvent chez Lucien, du rapport entre réalité et fiction, monde du lecteur et du narrateur, voire relation entre observateur et observé, puisqu'ici le narrateur a pu observer son lecteur, en miroir, par. 26 [30] :

[26] Et d'ailleurs il y a une autre merveille ( $\theta \propto u ̃ \mu \alpha)$ que j'ai observée dans le palais royal : un miroir (кátomтроv) très grand s'y trouve au-dessus d'un puits pas très profond. Si l'on descend dans le puits, on entend tout ce qui chez nous sur terre se dit, et si on regarde dans le miroir, ce sont toutes les cités et tous les peuples qu'on voit, comme si on se trouvait juste au-dessus d'eux : alors j'ai observé moi aussi mes parents et ma patrie entière, et si eux aussi me voyaient, je ne peux plus le dire avec certitude. Et celui qui ne croit pas qu'il en était ainsi, si un jour lui aussi se rend là-bas, il saura que je dis la vérité.

Cette organisation spatiale ressemble, par mise en abyme, à celle de la lecture, en surplomb par rapport à ce que le narrateur raconte et décrit, comme dans le dialogue du Charon où le nocher des Enfers et Hermès observent, du haut de montagnes réaménagées en gradins de théâtre, le monde humain et que le dieu psychopompe demande à son acolyte, dans les tous premiers mots du texte (par. 1) : Tí $\gamma \varepsilon \lambda \tilde{\alpha} s$,

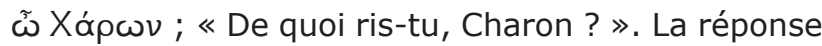
est que ce dernier désire « voir en quoi consiste la

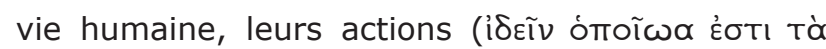

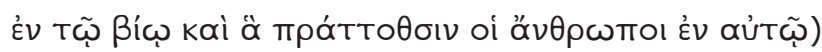
et ce dont la privation les fait se lamenter, quand ils descendent chez nous ». Du haut de la Lune, la
« comédie humaine » lucianesque fait du lecteur observateur un objet d'observation comique, à peu près comme les Sélénites, qu'observent le narrateur homodiégétique, pseudo-historien, et, par son intermédiaire, le lecteur de son livre, ou mieux, à l'époque, le spectateur-auditeur de sa performance sophistique [31]. Ainsi le lecteur humain devient-il paradoxalement et comiquement un sujet anthropologique comme d'autres, dans la Wunderkammer [32] spectaculaire des Histoires vraies qu'il croit d'abord dominer de son regard ethnocentré, comme le confirme ensuite la conclusion de l'épisode de l'île des Songes (II, 32-34) : « certains (songes) nous emmenaient aussi dans nos patries et nous montraient nos proches et nous ramenaient le jour-même ». Les humains sont «le rêve d'un songe »[33], plus comiques que pathétiques, ou d'autant plus pathétiques qu'ils sont ridicules, conformément au scepticisme typique de Lucien, souvent complété d'accents cyniques. Et tout cela concerne au plus haut point aussi les questions de genre et de sexualité, comme on le voit dans le reste des Histoires vraies.

\section{AILLEURS DANS LES HISTOIRES VRAIES : DIVERS JEUX DE GENRE ET SEXUALITÉ}

L'épisode des Sélénites, en particulier ses aspects sexuels, fait sens en écho à de multiples autres passages du même récit de voyage. On peut relever :

- les îles de femmes dangereusement séductrices, en début et fin de l'ouvrage. D'une part $(I, 8)$, les « femmes-vignes », une plante « prodigieuse » (TEpáotiov) au tronc robuste surmonté d'un corps de belle femme, à partir de la ceinture : deux compagnons de Lucien, ayant répondu à leurs avances,

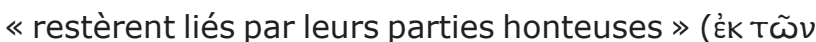

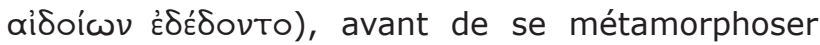
en végétaux porteurs de fruits; un scholiaste rapproche l'épisode des Lotophages, Odyssée, IX, 91. D'autre part (II, 46), les Onoskélées, « aux jambes d'ânesses », qui, « parées tout à fait à la manière de courtisanes et toutes belles et jeunes, traînent derrière elles des tuniques qui vont jusqu'aux pieds »,
[30] Voir FUSILLO 1988 et Ni MHEALLAIGH 2014, en particulier Fiction and reality: the worlds of the Moon, the whale and the Island of Dreams, p. 216-230.

[31] Sur le spectacle sophistique d'époque impériale, voir KoRENJAK 2000 et SCHMITZ 1997.

[32] Cette assimilation est empruntée à Ni MHEALLAIGH 2014, en lien avec la notion de « hyperreality » et les cabinets de curiosités et spectacles d'illusion, par exemple d'automates, dans la Rome impériale, « Conclusion. Fiction and the wonder-culture of the Roman empire », p. 261-277

[33] Selon l'idée mélancolique attestée de Pindare (Pythique, VIII, 95) à Rilke (p. ex. Requiem. Für eine Freudin). 
offrent aux voyageurs de passage I'hospitalité, pour les dévorer, comme les Sirènes évoquées aussi dans la préface. Le féminin est hybride et menaçant, comme la sexualité même, suivant une tradition culturelle grecque remontant à la figure de Pandora et au Monologue du misogyne (Sémonide fr. 7) [34].

- la figure d'Hélène (II, 8 et 25-27), sur l'île des Bienheureux, pour qui un procès oppose Thésée et Ménélas, avant que le fils d'un compagnon de Lucien, Kinyras, amoureux d'elle, ne l'enlève, avec son accord, pour s'enfuir à l'île de Phellô («De-Liège », II, 4) ou Tyroessa («Fromagère », II, 3), provoquant, non une autre guerre de Troie, mais un jugement du roi Rhadamanthe, qui le condamne à la façon des $\mu$ oıxoi de l'Athènes clas-

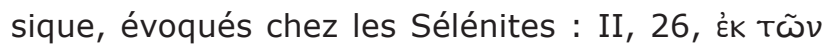

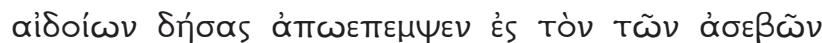

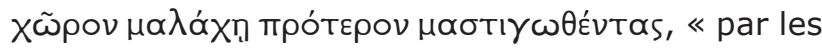
parties honteuses, il les fit attacher et les envoya au pays des impies, après les avoir fait fouetter de mauves ». Un détournement de la tradition homérique se retrouve, moins brutal, dans l'anecdote du courrier qu'Ulysse, déçu par Pénélope, fait parvenir à Calypso, qu'il aime encore (II, 35-36). La parodie des scènes amoureuses ou sexuelles des textes anciens, ici I'Iliade et l'Odyssée, utilise surtout le burlesque d'inversion.

- les mœurs sexuelles dans I'île des Bienheureux (II, 19), physiologiquement plus humaines que chez les Sélénites, mais de même issues de références culturelles, mythologiques, philosophiques. Selon Lucien, ces pratiques sont affaire d'opinion et représentation, relatives, qu'elles soient risibles ou non :

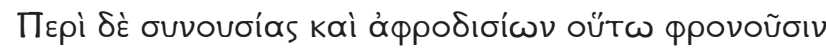
« et sur l'accouplement et les questions érotiques, voici ce qu'ils pensent. » La référence à l'impudeur des Cyniques est claire [35], ainsi que I'allusion à Hérodote sur les Indiens [36] : « ils s'unissent ouvertement aux yeux de tous à la fois aux femmes

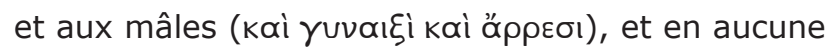
façon n'en conçoivent de honte ». De même pour I'allusion à la République, V, 457 sqq., étendue à toute la société : «Et les femmes sont communes à tous et personne n'envie son prochain : ils sont sur ce point tout à fait platoniciens ; et les garçons s'offrent à ceux qui veulent. »Ce dernier ajout est une pointe provocatrice, en référence aussi à I'Assemblée des femmes d'Aristophane et son utopie sexuelle. Cependant la cible est surtout l'ambiguïté de l'amour dit plus tard platonique, dont Socrate, chez Lucien, prétend sauvegarder la chasteté toute philosophique, contrairement à son évident comportement réel : « Socrate jurait certes que c'est en toute pureté qu'il avait commerce avec les jeunes gens, mais tous l'accusaient de parjure, et souvent, en tout cas, Hyacinthe et Narcisse avouaient, alors que lui niait. »

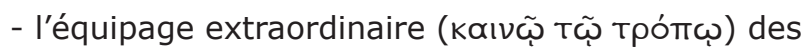
hommes qui sont « à la fois marins et navires», rencontrés juste avant l'île des femmes aux pieds d'ânesse (II, 45) : « couchés sur le dos dans l'eau ils dressaient leur sexe ( $\alpha i \delta$ oĩ) - ils en portent un grand, auquel ils attachent la voile et, tenant en main la bouline, suivant le souffle du vent, ils naviguaient. »

Ces passages évoquant sexe, genre et sexualité utilisent les procédés comiques à l'œuvre pour les Sélénites, et montrent aussi comment les Histoires vraies renvoient à trois mondes, à la fois distincts et comparables : la « réalité » (surtout du lecteur) ; la fiction fantastique imaginée par Lucien comme un spectacle intense, carnavalesque ; enfin l'univers que construit la lecture cultivée du lettré ou pepaideumenos, voire d'un public élargi, étant donné la trivialité de nombreuses références ici détournées [37]. Le comique, en particulier à propos du sexe, se fonde sur la cohabitation et parfois la confusion de ces trois mondes, par exemple quand le grand Socrate devient un dissimulateur honteux de comédie, l'utopie platonicienne une norme réalisée, ou I'obscénité monumentale un effet parfaitement naturel.

\section{EFFETS ET USAGES MODERNES ET CONTEMPORAINS : ENTRE AUTOCENSURE ET LECTURES INTÉRESSÉES}

On finira brièvement ce parcours par deux exemples significatifs de la réception moderne, en domaine francophone, de la description des mœurs sélénites, propre à montrer encore la relation complexe du sexe et du rire, surtout dans un dispositif aussi stratifié, contradictoire et ambivalent qu'une littérature de l'imaginaire à visée à la fois comique et sérieuse [38]. Le premier cas sera celui

[34] BRIAND 1998.

[35] Cf. chez Lucien, Le banquet ou les Lapithes, 16 et 46 .

[36] Référence que signale la scholie à Lucien, cf. Hérodote III, 101.

[37] Cette tripartition souple est inspirée de BouLOGNE 1996.

[38] Voir Bost-Fievet \& Provini 2014. 


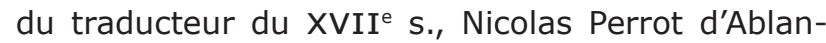
court, réputé pour ses «belles infidèles », selon la formule de Voltaire. Les passages qui nous occupent sont de ceux qu'il commente le plus pour justifier ce qu'on peut voir comme une autocensure : le contexte religieux, moral, social et donc littéraire n'est certes pas celui des Histoires vraies, dont sa traduction paraît en 1654 , et l'idée de décence a évolué. Sur les Sélénites, épouses avant vingt-cinq ans, époux après, Perrot précise : « Je ne dis pas qu'ils sont hommes après cela, pour ne pas insister sur des saletés, outre que cela s'entend assez. » Les Dendrites naissent simplement $d^{\prime} \ll$ un grand arbre charnu » et la comparaison « semblable à un phallus » est supprimée. Pour leurs organes génitaux postiches, il supprime aussi le syntagme que nous avons traduit par : «c'est avec cela qu'ils font leurs saillies et s'accouplent avec leurs conjoints. » Pour les mœurs des Bienheureux, l'amour en public disparaît ( « Je n'ai pas voulu mettre qu'ils les caressent devant tout le monde, ce qui est trop déshonnête ») et I'honneur de Socrate est sauvegardé (« Je n'ai pas voulu insister davantage sur cette saleté »). À l'inverse, pour la lettre d'Ulysse à Calypso, il invente une explication au fait que «Lucien » décachette la missive, " de peur que ce fourbe ne nous eût fait quelque supercherie » : «J'ai ajouté cela pour colorer cette action qui est indécente ». Enfin les navigateurs au phallus - mât se voient pourvus $d^{\prime} \ll$ un bâton entre les jambes, qui leur servait de mât » («ce qui est plus honnête que ce qui est et fait le même effet »). Cette dernière remarque prête à discussion, puisque ces infidélités au texte grec lui font perdre tout effet carnavalesque, tout « bas corporel » bakhtinien, sexualités différentes, ou encore l'ambivalence constitutive d'une fiction volontairement paradoxale. C'est le lien entre sexe et rire qui disparaît ici, au profit d'une euphémisation allégorisante que Perrot déploie d'ailleurs dans la suite qu'il a composée pour ces deux livres des Histoires vraies [39]. À la fin, par. 47, le narrateur formule une promesse (« ce qui s'est passé sur la terre de l'autre côté, je le raconterai dans les livres suivants ») que le scholiaste lui reproche de ne pas avoir tenue, et Perrot se charge d'un récit de voyage uniment sérieux et symbolique, où ni sexe ni rire ne sont en fait présents. Il est vrai que la réception de Lucien du $X V I^{e}$ au $X V I I I^{e} s$. est un processus complexe et contradictoire, bien étudié par ailleurs, de la condamnation par l'Église à la reconnaissance des Humanistes ou des Lumières, selon que ses accents sceptiques, voire cyniques, provoquent le rire ou I'horreur [40].

Le second exemple sera des plus actuels. Lucien peut être un garant reconnu, car antique, dans I'histoire d'un genre littéraire en quête de légitimité, à savoir la science-fiction, et ce alors que cette affiliation générique est au moins à débattre [41]. De même, la description des mœurs sexuelles et reproductrices des Sélénites est fréquemment utilisée, dans le cadre d'une « lecture intéressée » que nous avons qualifiée de queer en préambule, pour montrer que déjà dans I'Antiquité le mariage entre personnes de même sexe, voire la gestation pour autrui et la procréation médicalement assistée, semblaient possibles, au moins dans un monde lointain, et en tout cas n'étaient pas condamnées moralement puisqu'elles constituent la norme dans I'utopie lunaire. Un blog militant titrait même en 2009 : «le premier roman de science fiction de I'histoire de la littérature proposait déjà le mariage gay »[42]. À l'inverse, dans les mêmes années, pour les milieux réactionnaires, traditionnalistes et/ou d'extrême droite engagés en France contre le «mariage pour tous», les Histoires vraies, et notamment ce passage, serait emblématique de la «promotion de la débauche», issue de la «théorie du genre », à laquelle des «lobbies » voudraient faire succomber la « civilisation occidentale » [43]. On peut renvoyer à des plaintes de parents dénonçant le complot ourdi par l'Éducation nationale, quand notre ouvrage fut mis au programme des classes de Terminale littéraire, en grec, qui plus est avec en latin la Vie des douze Césars de Suétone
[39] Voir PERROT D'ABLANCOURT 1977 (1787) et 1988 (1654). On ne trouve plus ces omissions assumées dans la philologie moderne, même si les traductions y ont encore une vision très classique des questions de genre et sexualité, cf. GrimaL 1958.

[40] Pour un tableau général de la réception de Lucien, voir en dernier lieu BARTLEY 2009, et pour une analyse précise du rapport entre les Histoires vraies et Rabelais, CORREARD 2012.

[41] Voir RABAU 2014. Contra, par exemple, SWANSON 1976 et LARMOUR 1997. C'est aussi à RABAU 2015 que j'emprunte la notion de « lecture intéressée », appliquée notamment à des textes où les questions de genre et sexualité jouent un rôle important.

[42] Voir http://luclebelge.skynetblogs.be/tag/lucien + de+samosate. Suit, sur cette page internet, la référence aux Dialogues des courtisanes, pour l'homosexualité féminine.

[43] Voir http://lesalonbeige.blogs.com/my_weblog /2014/05/promotion-de-la-débauche-à-lécole-bompard -interroge-hamon.html 
et en français Les Mains libres d'Éluard [44]. Pour revenir à notre sujet, on remarquera que le lien entre rire (et fiction comique) et sexualité est ici totalement incompris, en particulier quand on ne voit pas que décrire des pratiques, surtout imaginaires, voire fantastiques, n'implique pas d'en faire l'éloge, mais plutôt d'opérer, en retour, une satire, d'abord divertissante, puis critique, relativiste et constructionniste, des pratiques et représentations culturelles les plus répandues, voire dominantes. On n'omettra pas non plus, plus souvent évoqué pour Aristophane que pour Lucien, le caractère d'ailleurs souvent possiblement réactionnaire du comique, ou au moins ambivalent sur le plan éthique et idéologique. Voilà donc le terme anachronique mais, espérons-le, maîtrisé [45], d'une expérience de décentrement, un détour par une œuvre et un auteur parmi les plus spectaculaires, séduisants et complexes de I'Antiquité grecque. On ne donnera pas plus que Lucien une réponse documentaire, historique, unique et complète à des questions que les Histoires vraies, affirmant que la « réalité » peut être une fiction et un jeu, une construction dynamique et toujours à contextualiser culturellement, n'essaient pas de traiter thématiquement, mais pragmatiquement, en faisant à la fois rire et penser, qu'il s'agisse des mœurs des Anciens, de leurs fictions et de ce que les modernes et contemporains tentent d'en faire.

[44] Voir à titre d'exemple une alerte aux parents catholiques, dont une citation suffira : « Le jeune bachelier qui a subi tout cela risque bien d'en sortir abîmé, sali, voire perverti dans sa mémoire, son imagination, sa sensibilité, son cœur, son intelligence, toute son âme » (http://coursprivecefop.org/images/2/22/ Alerte_aux_parents.pdf).

[45] Encore en référence à LoRAux 2005 (1993).

\section{BIBLIOGRAPHIE}

ANDERSON, Graham, 1976, Studies in Lucian's Comic Fiction, Leiden.

BARTLeY, Adam N., 2009, A Lucian for our times, Cambridge.

Billault, Alain (éd.), 1993/1994, Lucien de Samosate, Lyon.

Billault, Alain \& Marquis, Émeline (révis., annot.), 2015, Lucien de Samosate. Fuvres complètes, traduites par E. Chambry, Paris.

Boehringer, Sandra \& Sebillotte, Violaine (dir.), 2010, Hommes et femmes dans l'Antiquité grecque et romaine. Le genre : méthode et documents, Paris.

Boehringer, Sandra \& Briand, Michel (dir.), 2012, «Questions de genre et de sexualité dans l'Antiquité grecque et romaine », Lalies 32, p. 141-240.

Boehringer, Sandra \& Blondell, Ruby, 2014, « Leaina's Revenge: The Reception of Plato's Symposium in Lucian's Fifth Dialogue of the Courtesans », Arethusa, 47, p. 231-264.

Boehringer, Sandra \& Lorenzini, Daniele (dir.), 2016, Foucault, la sexualité, l'Antiquité, Paris.

BompaIRE, Jacques, 2000 (1958), Lucien écrivain, imitation et création, Paris.

BompaIRe, Jacques, 1998, Lucien. Euvres. Tome II : Opuscules 11-20.

Bost-Fievet, Mélanie \& Provini, Sandra (dir.), 2014, L'Antiquité dans l'imaginaire contemporain. Fantasy, science-fiction, fantastique, Paris.

Boulogne, Jacques, 1996, «Narrations véritables : miscellanées de toutes les hybridations imaginables », Uranie 6, Hybrides et hybridités, p. 81-101.

BRANHAM, Robert Bracht, 1989, Unruly Eloquence: Lucian and the Comedy of Traditions, Cambridge (Massachusetts). Branham, Robert Bracht, 2004, « Nietzsche's Cynicism: Uppercase or lowercase ? », dans Paul Bishop (éd.), Nietzsche and Antiquity. His Reaction and Response to the Classical Tradition, Rochester NY / Woodbridge, Suffolk, p. $170-181$. 
BRIAND, Michel, 1998, « Les origines de l'homme dans la lyrique archaïque grecque: fonctions et énonciations du mythe. A propos de Sémonide d'Amorgos, fr.7 (Diehl), Phocylide de Milet, fr.2 (Diehl), Alcman, fr.81 (Calame) et Pindare, Pythique IX, 41 sqq.», dans Jean-Michel Galy \& Antoine Thivel, Les origines de I'homme d'après les Anciens, Publications de la Faculté des Lettres de I'Université de Nice, n.s. n46, p. 55-70.

BRIAND, Michel, 2005, « Lucien et Homère dans les Histoires vraies : pratique et théorie de la fiction au temps de la Seconde Sophistique », Lalies 25, p.127-140.

BRIAND, Michel, 2007, «Les Dialogues des morts de Lucien, entre dialectique et satire : une hybridité générique fondatrice », Otrante 22, dans Ariane Eissen (dir.), Les dialogues des morts, p. 61-72.

BRIAND, Michel, 2008, «Les animaux de Lucien : fiction comique et (dé)-construction de l'humain », Culture classique et christianisme. Mélanges offerts à J. Bouffartigue, Paris, p. 315-329.

BrIAND, Michel, 2009, «L'Homère sophiste de Lucien ou les ambiguïtés d'une mimesis ironique », dans Glenn Most, Larry F. Norman, Sophie Rabau (éd.), Révolutions homériques, Pisa, p. 27-46.

BRIAND, Michel, 2010, « Le dialogue entre mythe et fiction : à propos du Dionysos de Lucien », dans Danièle Augier \& Charles Delattre (dir.), Mythe et fiction, Paris, p. 219-237.

BRIAND, Michel, 2012, «Pour un parallèle intempestif. Poètes norvégiennes contemporaines / grecques anciennes », Le Pan poétique des muses, « Poésie, Danse \& Genre », n²|Automne 2012, http://www.pandesmuses.fr

BRIAND, Michel, 2015a, «L'Homère paradoxal de Lucien. Un dialogue, entre imitation et satire », dans Sandrine Dubel, Anne-Marie Favreau-Linder \& Estelle Oudot (dir.), À l'école d'Homère. La culture des orateurs et des sophistes, Paris, p. $163-172$

BRIAND, Michel, 2015b, « Du contemporain dans la danse contemporaine ? », dans Cheryl F. Stock \& Patrick GermainThomas (éd.), Contemporising the past: envisaging the future, Proceedings of the 2014 WDA Global Summit, http://www. ausdance.org.au

BRIAND, Michel, 2016, « Rhetoric, liberties, and classical culture. From free speech (parrhêsia) to serio-comic (spoudogeloion) in Lucian of Samosata », Kaunas (Lit.), p. 185-198, https://eltalpykla.vdu.lt/handle/1/33191

BRIAND, Michel, 2017, «La transgénéricité des Histoires vraies: I'hybridation et la bigarrure comme modes de création, critique et connaissance », dans Émeline Marquis \& Alain Billault (dir.), Mixis. Le mélange des genres chez Lucien de Samosate, Paris, p. 71-89.

BRIAND, Michel, à paraître 2018, « Le queer (et le camp) antiquisant : Pierre et Gilles, Trajal Harrel et Cy Twombly », dans Fabien Bièvre-Perrin \& Élise Pampanay (éd.), Antiquipop. La référence à l'Antiquité dans la culture populaire contemporaine, Lyon.

Briand, Michel, Dubel, Sandrine \& Erssen, Ariane (éd.), 2017, Rire et dialogue, Rennes.

CAmerotto, Alberto, 1998, Le metamorfosi della parola. Studi sulla parodia in Luciano di Samosata, Pisa - Roma.

CAmerotro, Alberto, 2014, Gli occhi e la lingua della satira. Studi sull'eroe satirico in Luciano di Samosata, Milano - Udine.

Chantraine, Pierre, 1999, Dictionnaire étymologique de la langue grecque, Paris (1 $1^{\text {re }}$ éd. 1968-1980).

ColonnA, Vincent, 2004, Autofictions et autres mythomanies littéraires, Auch.

Correard, Nicolas, 2012, « Les Histoires vraies du Lucien français : de la poétique de l'incrédulité au regard moraliste du Quart Livre », Le Verger. Bouquet 1 Gargantua et le Quart Livre de Rabelais, http://cornucopia16.com/ blog/2014/07/21/janvier-2012-nicolas-correard-u-nantes-les-histoires-vraies-du-lucien-francais-de-la-poetique-de-lincredulite-au-regard-moraliste-du-quart-livre/

EISSen, Ariane \& Briand, Michel (éd.), 2014, Dialogue et théâtralité : interactions, hybridations, réflexivité. De Socrate à Derrida, / « Lucien (de Samosate) et nous », Cahiers du FoReLL en ligne : edel.univ-poitiers.fr/cahiersforell/ index.php?id=71

ERCOLANI, Andrea (hrsg.), 2002, Spoudaiogeloion. Form und Funktion der Verspottung in der aristophanischen Komödie, Stuttgart - Weimar.

Foucault, Michel, 1984, Histoire de la Sexualité 3. Le Souci de Soi, Paris.

FoucAult, Michel, 2009, Le courage de la vérité. Le gouvernement de soi et des autres II (Cours au Collège de France, 1984), Gallimard, Paris.

Fusillo, Massimo, 1988 « Le Miroir de la Lune », Poétique 73, p. 109-135.

GIANGRANDE, Lawrence, 1972, The Use of Spoudaiogeloion in Greek and Roman Literature, Den Hague - Paris.

Georgiadou, Aristoula \& LARMOUR, David, 1998, Lucian's Science Fiction Novel, Leiden.

GrIMAL, Pierre (prés., trad., annot.), 1958, Romans Grecs et Latins, Paris.

HALLIWELL Stephen, 2008, Greek Laughter. A Study of Cultural Psychology from Homer to Early Christianity, Cambridge. Helmer, Étienne, 2017, Diogène le cynique, Paris.

HOLMES, Brooke, 2012, Gender. Antiquity and its Legacy, Oxford.

HubBARD, Thomas K. (éd.), 2013, A Companion to Greek and Roman Sexualities, Malden (Massachussets).

JaY-RoberT, Ghislaine, 2014, «L'extravagance chez Aristophane et Lucien : une manière de penser », dans PaulAugustin Deproost (éd.), Extravagances. Écarts et normes dans les textes grecs et latins, Paris, p. 21-37.

KORENJAK, Martin, 2000, Publikum und Redner. Ihre Interaktionen in der sophistischen Rhetorik der Kaiserzeit, München. LACAZE, Guy (intr., trad. et notes), 2003, Lucien. Histoires vraies et autres œuvres, Paris.

LAIRD, Andrew, 2003, « Fiction as a Discourse of Philosophy in Lucian's Verae Historiae », dans Stelios Panayotakis, Maaike Zimmerman \& Wytse Keulen (éd.), The Ancient Novel and Beyond, Leiden, p. 115-127.

LAMI, Alessandro \& Maltomini, Franco, 1990, Dialoghi di dei e di cortigiane, Milano. Un antico postmoderno, p. 1-35. LARMOUR, David H. J., 1997, « Sex with Moonmen and Vinewomen: The Reader as Explorer in Lucian's Vera Historia », Intertexts 1.2, p. 131-146. 
Ligota, Christopher \& Panizza, Letizia (éd.), 2007, Lucian of Samosata Vivus and Redivivus, London - Turin.

LORAUX, Nicole, 2005 (1993), « Éloge de I'anachronisme en histoire », Le genre humain n 27, 1993, 23-39, repris dans Les Voies traversières de Nicole Loraux. Une helléniste à la croisée des sciences sociales, numéro commun Espaces Temps Les Cahiers n 87-88 et CLIO, Histoire, Femmes et Sociétés, p. 128-139.

Lüthe, Rudolf, 2013, Skepsis, Melancholie, Ironie. Facetten einer philosophischen Orientierung in der postmodernen Kultur, Berlin.

Masterson, Mark, Rabinowitz, Nancy \& Robson, James (éd.), 2014, Sex in Antiquity : Exploring Gender and Sexuality in the Ancient World, Oxford - New York.

MATZNER, Sebastian, 2016, «Queer Unhistoricism : Scholars, Metalepsis, and Interventions of the Unruly Past », dans Shane Butler (éd.), Deep Classics. Rethinking Classical Reception, London - New York, p. 179-201.

Mestre, Francesca \& Gomez, Pilar (éd.), 2010, Lucian of Samosata. Greek writer and Roman citizen, Barcelona.

MOINEAU, Jean-Claude, 2016, Queeriser l'art, Paris.

Möllendorff, Peter von, 2000, Auf der Suche nach der verlogenen Wahrheit: Lukians Wahre Geschichten, Tübingen. Narbonne, Jean-Marc, 2016, Antiquité critique et modernité. Essai sur le rôle de la pensée critique en Occident, Paris. Ni Mheallaigh, Karen, 2014, Reading Fiction with Lucian. Fakes, Freaks and Hyperreality, Cambridge.

OzanAM, Anne-Marie (trad., annot.), 2009, Lucien. Voyages extraordinaires, Paris.

Perrot d'Ablancourt, Nicolas (prés. et trad.), 1988, Lucien. Histoire véritable, Arles (1e éd. 1654).

Perrot d'Ablancourt, Nicolas, 1977, Lucien, Histoire Véritable, traduite et continuée par P.d'Ablancourt, 2 vols., Nancy (éd. de 1787).

RABAU, Sophie, 2014, « Pourquoi dit-on que Lucien est un auteur de Science-Fiction ? », dans EISSEN \& BRIAND 2014.

RABAU, Sophie, 2015, « Sabotage littéraire 1 à 4. Lecture intéressée », Vacarme 70-73 (www.vacarme.org).

RenNes, Juliette (dir.), 2017, Encyclopédie critique du genre. Corps, sexualité, rapports sociaux, Paris.

Schмrтz, Thomas, 1997, Bildung und Macht. Zur sozialen und politischen Funktion der zweiten Sophistik in der griechischen Welt der Kaiserzeit, München.

Scolan, Yannick, 2017, Le Convive et le Savant. Sophistes, rhéteurs, grammairiens et philosophes au banquet de Platon à Athénée, Paris.

Setembrini, Luigi (trad.) \& Fusaro, Diego (intr., note e appariti), 2007, Luciano di Samosata. Tutti gli Scritti, Milano, 2007.

SluIter, Ineke \& Rosen, Ralph M., 2004, Free Speech in Classical Antiquity, Leiden.

Swanson, Roy Arthur, 1976, «The True, the False, and the Truly False: Lucian's Philosophical Science Fiction », Science Fiction Studies 10.3.

WhItMARSH, Tim, 2011, Greek Literature and the Roman Empire. The Politics of Imitation, Oxford, chap. V Satirizing Rome: Lucian, p. 247-293.

WhitmaRsh, Tim, Battling the Gods. Atheism in the Ancient World, London, 2016. chap. 15 Imagine, p. 215-230. 